\title{
BMJ
}

\section{Effect of prolonged and exclusive breast feeding on risk of allergy and asthma: cluster randomised trial}

\author{
Michael S Kramer, James McGill professor; and scientific director," Lidia Matush, chief , 2 \\ Irina Vanilovich, senior scientist, ${ }^{3}$ Robert Platt, associate professor; and investigator and associate director, ${ }^{4}$ \\ Natalia Bogdanovich, senior scientist, ${ }^{3}$ Zinaida Sevkovskaya, senior scientist, ${ }^{3}$ Irina Dzikovich, senior \\ scientist,, Gyorgy Shishko, director , ${ }^{3}$ Bruce Mazer,, associate professor; and head, ${ }^{5}$ for the Promotion of \\ Breastfeeding Intervention Trial (PROBIT) Study Group
}

$\overline{1}$ Departments of Pediatrics and of Epidemiology and Biostatistics, McGill University Faculty of Medicine; and Institute of Human Development and Child and Youth Health, Canadian Institutes of Health Research (CIHR), Montreal, Canada

${ }^{2}$ Maternal and Child Health Department, Belarussian Ministry of Health

${ }^{3}$ Belarussian Maternal and Child Health Research Institute

${ }^{4}$ Departments of Pediatrics and of Epidemiology and Biostatistics, McGill University; and IS/IT, McGill University Health Centre Research Institute, Montreal

${ }^{5}$ Department of Pediatrics, McGill University; and Division of Pediatric Allergy and Clinical Immunology, Montreal Children's Hospital, McGill University Health Centre

Correspondence to: M S Kramer, Montreal Children's Hospital, 2300 Tupper Street (Les Tourelles),

Montreal, Quebec H3H 1P3

michael.kramer@mcgill.ca

do:10.1136/bmi.39304.464016.AE

\section{ABSTRACT}

Objective To assess whether exclusive and prolonged breast feeding reduces the risk of childhood asthma and allergy by age 6.5 years.

Design Cluster randomised trial.

Setting 31 Belarussian maternity hospitals and their affiliated polyclinics.

Participants A total of 17046 mother-infant pairs were enrolled, of whom 13889 (81.5\%) were followed up at age 6.5 years.

Intervention Breastfeeding promotion intervention modelled on the WHO/UNICEF baby friendly hospital initiative.

Main outcome measures International study of asthma and allergies in childhood (ISAAC) questionnaire and skin prick tests of five inhalant antigens.

Results The experimental intervention led to a large increase in exclusive breast feeding at 3 months $(44.3 \% \mathrm{~V}$ $6.4 \% ; P<0.001)$ and a significantly higher prevalence of any breast feeding at all ages up to and including 12 months. The experimental group had no reduction in risks of allergic symptoms and diagnoses or positive skin prick tests. In fact, after exclusion of six sites (three experimental and three control) with suspiciously high rates of positive skin prick tests, risks were significantly increased in the experimental group for four of the five antigens.

Conclusions These results do not support a protective effect of prolonged and exclusive breast feeding on asthma or allergy.

Trial registration Current Controlled Trials ISRCTN37687716.

\section{INTRODUCTION}

Whether breast feeding protects against the development of allergy and asthma has been frequently studied and hotly debated for more than 70 years. ${ }^{1-8}$ Research findings indicating a beneficial effect have been most consistent for atopic eczema during infancy, but the evidence on asthma and other atopic outcomes (including hay fever, food allergies, and positive skin tests) has been far more mixed. Some studies have reported greater degrees of protection with more exclusive and prolonged breast feeding, ${ }^{9-12}$ and several have noted a larger protective effect in children prone to atopy. ${ }^{45}$ Other studies, however, have reported no reduction in risk or even an increase in risk with breast feeding. ${ }^{13-20}$

A variety of methodological problems are likely to have affected the evidence base on infant feeding and allergic disease. Virtually all of the evidence is based on observational studies. Case-control studies are prone to recall bias, which may be systematic if the people who ascertain the previous feeding history are aware of the case versus control status of the study participants. Cohort studies, on the other hand, are prone to biased assessment of outcomes when observers are not blinded to the previous infant feeding history, a particularly important problem for outcomes such as atopic eczema, hay fever, and asthma, for which no definitive laboratory measures are available to provide a "gold standard" for diagnosis. Misclassification of infant feeding history, particularly with respect to differences in degree or duration of breast feeding, is always a problem in studying the health effects of breast feeding but is even more problematic in studies of atopic disease. This is because hypersensitivity reactions in particular and atopic disease in general may not show the dose-response effects seen with other outcomes related to infant feeding and may be affected not only by the quantity of exposure but also by its frequency and timing. One recent study has even reported evidence of reverse causality, whereby early signs of atopic disease led to earlier discontinuation of exclusive breast feeding. ${ }^{21}$ Finally, publication bias may well have affected the evidence base, with selective submission and publication of studies with positive findings (that is, showing that breast feeding is protective).

One potential solution to these methodological problems is a randomised controlled trial. Although randomising healthy mothers and infants to breast feeding versus formula feeding is infeasible and probably unethical, randomising them to an intervention that 


\begin{tabular}{|c|c|c|}
\hline Variable & $\begin{array}{l}\text { Experimental group } \\
\qquad(n=7108)\end{array}$ & $\begin{array}{c}\text { Control group } \\
(n=6781)\end{array}$ \\
\hline \multicolumn{3}{|l|}{ Maternal age (years): } \\
\hline$<20$ & $1017(14.3)$ & $897(13.2)$ \\
\hline $20-34$ & $5783(81.4)$ & $5598(82.6)$ \\
\hline$\geq 35$ & $308(4.3)$ & $286(4.2)$ \\
\hline \multicolumn{3}{|l|}{ Maternal education: } \\
\hline Incomplete secondary & $310(4.4)$ & $202(3.0)$ \\
\hline Complete secondary & 2441 (34.3) & $2011(29.7)$ \\
\hline Advanced secondary or partial university & 3395 (47.8) & $3693(54.5)$ \\
\hline Complete university & $962(13.5)$ & $875(12.9)$ \\
\hline \multicolumn{3}{|l|}{ Older children living in household: } \\
\hline 0 & $4176(58.8)$ & 3695 (54.5) \\
\hline 1 & $2368(33.3)$ & $2450(36.1)$ \\
\hline$\geq 2$ & $564(7.9)$ & $636(9.4)$ \\
\hline Positive family history of atopy & $359(5.1)$ & $226(3.3)$ \\
\hline Maternal smoking during pregnancy & $184(2.6)$ & $108(1.6)$ \\
\hline Male child & $3653(51.4)$ & $3528(52.0)$ \\
\hline Mean (SD) birth weight (g) & $3440(418)$ & 3441 (423) \\
\hline
\end{tabular}

promotes breast feeding is both feasible and ethical. One potential strategy would be to intervene prenatally to increase the proportion of mothers who start breast feeding, but such a strategy poses logistical problems of reaching and influencing women during or even before pregnancy. An alternative and more practical strategy is to promote exclusivity and duration of breast feeding among those mothers who have already decided to start breast feeding. This is the strategy we used in designing and implementing the promotion of breastfeeding intervention trial (PROBIT), a cluster randomised trial in the Republic of Belarus. ${ }^{22}$ In this paper, we describe the methods and results of measures of allergic symptoms and diagnoses and skin prick tests after 6.5 years of follow-up among Belarussian children enrolled in PROBIT.

\begin{tabular}{ll}
\hline Table $2 \mid$ Audit results & $\mathrm{K}(95 \% \mathrm{Cl})$ \\
\begin{tabular}{ll} 
ISAAC questions $(\mathrm{n}=190)$ & $0.77(0.61$ to 0.92$)$ \\
\hline Ever had wheezing & $0.74(0.59$ to 0.90$)$ \\
\hline Wheezing in past 12 months & $0.66(0.52$ to 0.81$)$ \\
\hline Ever had asthma & $0.88(0.73$ to 1.00$)$ \\
\hline Ever had hay fever symptoms & $0.92(0.77$ to 1.00$)$ \\
\hline Hay fever symptoms in past 12 months & $0.53(0.39$ to 0.67$)$ \\
\hline Recurrent itchy rash & $0.50(0.36$ to 0.63$)$ \\
\hline Ever had eczema & $0.78(0.49$ to 1.00$)$ \\
\hline Skin prick test antigens ( $=54)$ & $0.59(0.30$ to 0.87$)$ \\
\hline House dust mite & $0.49(0.21$ to 0.78$)$ \\
\hline Cat & $0.94(0.65$ to 1.00$)$ \\
\hline Birch pollen & $0.55(0.26$ to 0.84$)$ \\
\hline Mixed northern grasses & \\
\hline Alternaria & \\
\hline ISAAC=international study of asthma and allergy in childhood.
\end{tabular} \\
\hline
\end{tabular}

\section{METHODS}

The detailed methods of PROBIT and the results during the first year of follow-up have been previously reported. ${ }^{22}$ The units (clusters) of randomisation were maternity hospitals and one affiliated polyclinic (outpatient clinic where children are followed for well child and illness care) for each hospital, with double randomisation based on both a random numbers table and a coin flip. ${ }^{22}$ The experimental intervention was based on the baby friendly hospital initiative, which was developed by the World Health Organization (WHO) and the United Nations Children's Fund (UNICEF) to promote and support breast feeding, particularly among mothers who have chosen to start breast feeding. ${ }^{23}$ The control maternity hospitals and polyclinics continued the practices and policies in effect at the time of randomisation. The trial results are based on a total of 17046 healthy breastfed infants from 31 maternity hospitals/polyclinics; all were born at term in 1996-7, weighed at least $2500 \mathrm{~g}$, and were enrolled during their postpartum stay. ${ }^{22}$ To our knowledge, PROBIT is the largest randomised trial ever done in the area of human lactation. It conforms to the CONSORT recommendations for the design, analysis, and reporting of cluster randomised trials. ${ }^{24}$

As previously reported, ${ }^{22}$ the two randomised groups were similar in baseline sociodemographic and clinical variables, including maternal age, education, number of other children at home, proportion who had breast fed a previous child for at least three months, family history of atopy, caesarean delivery, maternal smoking during pregnancy, birth weight, gestational age, and five minute Apgar score. The experimental intervention led to a substantial difference in the duration of any breast feeding that was maintained throughout the first year of follow-up: $72.7 \%$ versus $60.0 \%$ were still breast feeding at 3 months, $49.8 \%$ versus $36.1 \%$ at 6 months, $36.1 \%$ versus $24.4 \%$ at 9 months, and $19.7 \%$ versus $11.4 \%$ at 12 months in the experimental and control groups. In addition, the prevalence of exclusive breast feeding was sevenfold higher in the experimental group at 3 months $(43.3 \%$ v $6.4 \% ; \mathrm{P}<0.001)$, although low in both groups at 6 months $(7.9 \% v 0.6 \% ; \mathrm{P}=0.01) .^{22}$

One paediatrician in each of 24 of the 31 polyclinics did follow-up interviews and examinations at age 6 . 5 years from December 2002 to April 2005; in the remaining seven high volume clinics, follow-up visits were shared by two paediatricians. Allergy symptoms and diagnoses were ascertained with the international study of asthma and allergy in childhood (ISAAC) questionnaire, which had already been translated and validated by the ISAAC investigators. ${ }^{25}$ In addition, the paediatricians did skin prick tests to five antigens (Allergy Canada): house dust mite, cat, birch pollen, mixed northern grasses, and Alternaria. Saline was included as a negative control and histamine (1 mg/ $\mathrm{ml}$ ) as a positive control. The criteria for a positive result were a mean wheal $\geq 3 \mathrm{~mm}$ or flare $\geq 10 \mathrm{~mm}$, calculated as the mean of the longest diameter and orthogonal diameter after subtracting the mean of these 
Table3 Positive skin prick test wheal remeasurements $(n=119)$. Values are intraclass correlation coefficients ( $95 \%$ confidence intervals)

\begin{tabular}{lll} 
Antigen & Longest diameter & Orthogonal diameter \\
House dust mite & $0.96(0.95$ to 0.98$)$ & $0.96(0.94$ to 0.97$)$ \\
\hline Cat & $0.93(0.90$ to 0.95$)$ & $0.86(0.81$ to 0.94$)$ \\
\hline Birch pollen & $0.94(0.91$ to 0.95$)$ & $0.97(0.96$ to 0.98$)$ \\
\hline Mixed northern grasses & $0.95(0.92$ to 0.96$)$ & $0.98(0.98$ to 0.99$)$ \\
\hline Alternaria & $0.94(0.91$ to 0.96$)$ & $0.92(0.89$ to 0.95$)$ \\
\hline
\end{tabular}

diameters for the saline control. A negative test result required a positive histamine test: mean diameter with histamine minus mean diameter with saline $\geq 3 \mathrm{~mm}$ for wheal or $\geq 5 \mathrm{~mm}$ for flare. A line in marker pen was drawn around each flare and wheal and transferred to paper tape, which was then stuck on to the data forms sent to the PROBIT data centre in Minsk.

Because blinding of paediatricians to the experimental versus control assignment was not feasible, we randomly selected five children per paediatrician $(n=38)$ for audit, for a total of 190 audited children. To ensure that all children followed up in PROBIT II were eligible to be audited, we did the audits after completion of follow-up visits, a mean of 17.7 (range 5.3-32.6) months after the initial polyclinic visit. One of the Minsk based paediatrician collaborators (LM, ZS, ID, or GS) did the audits; they were blind to the responses to the ISAAC questionnaire and results of the skin prick tests obtained at the initial clinic visit but not to the experimental versus control treatment allocation. We used the $\kappa$ coefficient (and its 95\% confidence interval) to assess inter-observer agreement. As a further data quality control check, we remeasured the wheal and flare results recorded on transfer to paper tape on a randomly selected sample of $1 \%(n=119)$ of the children with positive test results. We used the intraclass correlation coefficient (and its 95\% confidence interval) to quantitate the agreement between the two measurements.

We based all statistical analyses on intention to treat. We used the GLIMMIX procedure in SAS (version $8.2)$ to analyse differences in outcome between the experimental and control groups. This is a multilevel statistical modelling procedure that uses penalised quasi-likelihood for generalised linear mixed models and accounts for the clustered randomisation. ${ }^{26}$ The

\begin{tabular}{|c|c|c|c|c|}
\hline Question & $\begin{array}{l}\text { Experimental group } \\
\qquad(\mathrm{n}=7101)\end{array}$ & $\begin{array}{l}\text { Control group } \\
(n=6763)\end{array}$ & ICC & $\begin{array}{c}\text { Cluster adjusted } \\
\text { odds ratio* }(95 \% \mathrm{Cl})\end{array}$ \\
\hline Ever had wheezing & $778(11.0)$ & $651(9.6)$ & 0.03 & 1.1 (0.6 to 1.8$)$ \\
\hline Wheezing in past 12 months & $238(3.4)$ & $188(2.8)$ & 0.01 & $1.0(0.7$ to 1.6$)$ \\
\hline Ever had asthma & $97(1.4)$ & $68(1.0)$ & 0.00 & $1.2(0.7$ to 1.9$)$ \\
\hline Ever had hay fever symptoms & $384(5.4)$ & $257(3.8)$ & 0.02 & $1.1(0.6$ to 1.9$)$ \\
\hline $\begin{array}{l}\text { Hay fever symptoms in past } \\
12 \text { months }\end{array}$ & $262(3.7)$ & $192(2.8)$ & 0.01 & $1.0(0.6$ to 1.8$)$ \\
\hline Recurrent itchy rash & $350(4.9)$ & $241(3.6)$ & 0.02 & 1.3 (0.7 to 2.2$)$ \\
\hline Ever had eczema & $69(1.0)$ & $72(1.1)$ & 0.00 & $1.0(0.5$ to 1.8$)$ \\
\hline
\end{tabular}

ICC=intraclass correlation coefficient.

*For experimental group versus control group. procedure estimates odds ratios and 95\% confidence intervals for dichotomous outcomes, thus permitting inference at the level of the individual child, rather than at the level of the cluster (maternity hospital and polyclinic). The cluster adjusted differences presented in the text and tables are very similar to those obtained from a multivariate model that adjusts for stratum level variables (geographic region (west $v$ east) and urban $v$ rural location), as well as the following individual level covariates: age at follow-up, sex, birth weight, family history of atopy, pet ownership, contact with farm animals, and previous exposure to probiotics (multivariate results available on request). We did not impute missing data. Finally, because several previous studies have suggested that breast feeding is more protective in infants with a positive first degree (parent or sibling) family history of atopy, ${ }^{45}$ we also analysed mixed models that included terms for family history and a multiplicative interaction term for family history and treatment.

\section{RESULTS}

A total of 13889 children were seen in follow-up for PROBIT II, representing $81.5 \%$ of the 17046 originally randomised. Of the 3157 children randomised but not followed up, 88 had died, 2938 were lost to followup, and 131 were unable or unwilling to come for their PROBIT II visit. Follow-up rates were similar in the experimental $(80.2 \%)$ and control $(82.9 \%)$ polyclinics but varied considerably by polyclinic - from $56.1 \%$ at one of the Minsk polyclinics to $94.6 \%$ at Klimovichi, a small rural based polyclinic. The mean age at followup was 6.6 (SD 0.3) years. As shown in table 1, the children followed up in the experimental and control groups were similar in baseline characteristics, with small differences paralleling those seen (and previously reported ${ }^{22}$ ) at randomisation.

Table 2 shows the audit results. The data shown are the $\kappa$ coefficients (and their 95\% confidence intervals) between the results at the initial clinic visit and the results at the audit visit. These $\kappa$ values are high with respect to wheezing and hay fever symptoms and moderate for reported diagnosis of asthma or symptoms and diagnosis of atopic eczema. Concordance was high for the skin prick test results, but only $54(28 \%)$ of the 190 audited children (or their parents) agreed to the repeat skin tests. The results of the random skin prick test wheal remeasurements indicated exceptionally high intraclass correlation coefficients for both the longest diameter and orthogonal diameter for all five antigens tested (table 3 ).

Table 4 shows the trial results for the ISAAC questionnaire, the number and proportion of those with positive responses in the experimental and control groups, the intraclass correlation coefficients reflecting the degree of within polyclinic clustering, and the cluster adjusted odds ratios and their $95 \%$ confidence intervals. The results showed a very low degree of clustering - that is, very little tendency for mothers of children attending the same polyclinic to respond more similarly to each other than those of children attending 
Table $5 \mid$ Skin prick test results. Values are numbers (percentages) positive unless stated otherwise

\begin{tabular}{lcccc} 
Antigen & $\begin{array}{c}\text { Experimental group } \\
(\mathrm{n}=5551)\end{array}$ & $\begin{array}{c}\text { Control group } \\
(\mathrm{n}=5595)\end{array}$ & ICC & $\begin{array}{c}\text { Cluster adjusted } \\
\text { odds ratio* }(95 \% \mathrm{Cl})\end{array}$ \\
House dust mite & $805(14.5)$ & $603(10.8)$ & 0.14 & $1.1(0.5$ to 2.4) \\
\hline Cat & $648(11.7)$ & $491(8.8)$ & 0.20 & $1.2(0.5$ to 2.8) \\
\hline Birch pollen & $526(9.5)$ & $393(7.0)$ & 0.18 & $1.2(0.5$ to 2.9) \\
\hline Mixed northern grasses & $712(12.8)$ & $491(8.8)$ & 0.17 & $1.0(0.5$ to 2.3) \\
\hline Alternaria & $480(8.6)$ & $340(6.1)$ & 0.18 & $1.5(0.5$ to 4.4) \\
\hline$\geq 1$ positive & $1496(27.0)$ & $1013(18.1)$ & 0.19 & $1.2(0.5$ to 2.6) \\
\hline
\end{tabular}

ICC=intraclass correlation coefficient.

*For experimental group versus control group.

different polyclinics. Most of the symptoms and diagnoses were slightly more prevalent in the experimental group than in the control group, but the cluster adjusted odds ratios in the experimental versus control groups were close to unity for all of the symptoms and diagnoses.

Table 5 shows the skin prick test results. Of the 13889 PROBIT children seen at follow-up, 11772 $(85 \%)$ agreed to the skin prick tests, of whom 11146 $(95 \%)$ had valid results, as judged by the criterion of a positive test to histamine (see methods). Positive skin test results were slightly but consistently more frequent in the experimental group for all five test antigens, although none of the differences was statistically significant. We also analysed the combination of one or more positive skin prick tests plus each of the allergic symptoms and diagnoses from the ISAAC questionnaire. The cluster adjusted odds ratios were very close to 1.0 for all of these combinations (data not shown).

As suggested by the high intraclass correlation coefficients and the wide confidence intervals for the odds ratios (table 5), skin prick test results were extremely variable and highly clustered among the 31 polyclinics. In fact, the range among the 31 clinics was from $4 \%$ to $85 \%$ with one or more positive tests. Six of the polyclinics had positivity rates of $\geq 10 \%$ to each of the five test antigens, which was considerably higher than those at the 25 other polyclinics and inconsistent with the low prevalence of atopic symptoms and diagnoses as revealed by the ISAAC questionnaire. Despite repeated questioning, the paediatricians indicated no problems with the technique used for administering

Table 6 | Results of sensitivity analysis for skin prick test results. Values are numbers (percentages) positive unless stated otherwise

\begin{tabular}{lcccc} 
Antigen & $\begin{array}{c}\text { Experimental group } \\
(\mathrm{n}=4100)\end{array}$ & $\begin{array}{c}\text { Control group } \\
(\mathrm{n}=4906)\end{array}$ & ICC & $\begin{array}{c}\text { Cluster adjusted odds } \\
\text { ratio* }(95 \% \mathrm{Cl})\end{array}$ \\
House dust mite & $504(12.3)$ & $299(6.1)$ & 0.04 & $2.0(1.2$ to 3.4) \\
\hline Cat & $347(8.5)$ & $182(3.7)$ & 0.05 & $2.1(1.1$ to 3.9) \\
\hline Birch pollen & $273(6.7)$ & $125(2.5)$ & 0.03 & $2.3(1.3$ to 4.1) \\
\hline Mixed northern grasses & $369(9.0)$ & $209(4.3)$ & 0.06 & $1.5(0.8$ to 2.8) \\
\hline Alternaria & $258(6.3)$ & $77(1.6)$ & 0.05 & $3.5(1.6$ to 7.7$)$ \\
\hline$\geq 1$ positive & $929(22.7)$ & $579(11.8)$ & 0.07 & $2.0(1.1$ to 3.4)
\end{tabular}

ICC=intraclass correlation coefficient.

*For experimental group versus control group. the skin prick test, nor were any problems detected during monitoring visits carried out soon after data collection had begun. Redefining positive skin prick tests on the sole basis of the size of the wheal did not reduce the high positivity rates in the six discrepant clinics, nor did it uncover any protective effects of the experimental intervention (data available on request).

We did a sensitivity analysis $(n=9006)$ after excluding the six polyclinic sites with high rates of positive skin prick test results (three of these polyclinics were in the experimental group and three were in the control group). Table 6 shows the results of the sensitivity analysis. Intraclinic clustering of skin prick test results was reduced substantially. The proportions of positive test results were considerably lower in both the experimental and control groups, yet the differences between the two groups increased, with significantly elevated odds ratios in the 2-3 range for all but one antigen (mixed northern grasses).

Statistical models with interaction terms showed no evidence that the overall effects of the intervention on allergic symptoms and diagnoses were modified by family history of atopy, except for a history of ever wheezing, for which the experimental intervention increased the risk to a greater extent among those with a positive family history $(\mathrm{P}=0.005)$. For the skin prick tests, we found a significant interaction in the same direction (greater risk increase in those with a positive family history) only for mixed northern grasses $(\mathrm{P}=0.02)$.

Finally, we also examined the observational associations between the duration and exclusivity of breast feeding and the prevalence of allergic symptoms and diagnoses and skin prick test results. For allergic symptoms and diagnoses, we found borderline significant reductions in history of eczema both with more prolonged any breast feeding and with more prolonged exclusive breast feeding $(\mathrm{P}=0.08$ for both associations, based on $\chi^{2}$ tests for trend). Associations were stronger and in the opposite direction for skin prick test results, especially after elimination of the six sites with high rates of positive tests; we found highly significant increases in positive skin prick test results with exclusive breast feeding for 3 to $<6$ months and $\geq 6$ months versus $<3$ months for house dust mite, cat, birth pollen, mixed northern grasses, and Alternaria $(\mathrm{P}<0.001$ for all five antigens, based on $\chi^{2}$ tests for trend).

\section{DISCUSSION}

The results from this large cluster randomised trial indicate that the experimental intervention to promote breast feeding did not reduce the risk of asthma, hay fever, or eczema at age 6.5 years despite large increases in the duration and exclusivity of breast feeding; nor did the intervention succeed in reducing the prevalence of positive skin prick tests. We observed high inter-paediatrician variability (and consequently wide confidence intervals around the adjusted odds ratios in results of skin prick tests. After exclusion of six polyclinics with suspiciously high rates of positive skin prick test results, the relative odds of positive skin 


\section{WHAT IS ALREADY KNOWN ON THIS TOPIC}

Evidence is conflicting as to whether prolonged and exclusive breast feeding increases, decreases, or has no effect on the risks of asthma and allergy

All of the available evidence is based on observational studies

\section{WHAT THIS STUDY ADDS}

Prolonged and exclusive breast feeding had no protective effect on allergic symptoms and diagnoses or on positive skin prick tests

prick tests were twofold to threefold higher in the experimental group than in the control group.

These results conflict with some previous studies suggesting that prolonged and exclusive breast feeding reduces the risk of asthma, other allergic diseases, and atopy skin tests. ${ }^{4-69-1227}$ On the other hand, as discussed in the introduction, the evidence is far from uniform, and several recent studies have even found breast feeding (or more exclusive and prolonged breast feeding) to be associated with increased risks of these outcomes. ${ }^{15-20}$ Some investigators have found stronger protective effects of breast feeding in offspring with a positive family history of atopy, ${ }^{45}$ but we did not. The prevalences of all allergic symptoms and diagnoses were lower among PROBIT children than are customarily seen in Western industrialised countries but similar to those previously reported from Eastern Europe. ${ }^{28}$ Caution is therefore advised in extrapolating our results to settings where atopic diseases occur more frequently.

The absence of a protective effect against eczema also conflicts with our previous findings based on follow-up during infancy. ${ }^{22}$ The extremely low reported histories of eczema at 6.5 years (table 4), however, are likely to be gross underestimates. These histories may reflect the absence of an eczema "label" transmitted to the parents but almost certainly constitute a less objective result than that previously reported from PROBIT, in which the diagnosis was based on a standard algorithm using physical findings and symptom histories documented by paediatricians on six occasions during the first 12 months of life. ${ }^{22}$

The finding of exceedingly high rates of positive skin tests at six of the study sites, equally divided between experimental and control groups, is not easily explained. Something systematic clearly occurred at these sites, leading to our decision to eliminate them in the sensitivity analysis. These six study sites had no obvious links. They were dispersed geographically; two were in Vitebsk region (north), and one each were in Grodno region (northwest), Gomel region (south), Mogilev region (east), and Minsk region (centre). Two were in urban areas (Retchitsa and Mogilev), whereas the remaining four were rural (Glubokoe, Berestovitsa, Dokshitsy, and Minsk Regional). More importantly, however, the six sites did not have higher prevalences of allergic symptoms and diagnoses (on the basis of the ISAAC questionnaire) than the 25 sites with much lower skin prick test positivity rates. Skin testing is influenced by the technique of the tester, the device used for administration, the potency of the extracts, the spacing between the antigens, the position of the arm used for placement, and even the time of day. ${ }^{2930}$ All testers were trained by the same investigator (BM), all centres were provided with the same testing equipment, and the extracts were prepared from the same lots for all participants. Other possible explanations include failure to wipe the testing needle after histamine or antigens, contamination of the extract preparation with histamine, trauma with the skin prick test needle, and placement of the test antigens too closely together. The small number $(\mathrm{n}=54)$ of children with repeat skin tests at the audit, and the auditing paediatricians' use of the polyclinics' antigen solutions, prevented use of the audit data to confirm or refute these possibilities.

Given that we found significantly increased risks of positive skin prick tests in the experimental group only after excluding the six suspect polyclinics, we cannot be confident that the experimental intervention actually caused the increased risks. We feel on safer ground in inferring no reduction in risk. Given these results based on a large randomised trial and the inconsistent benefits reported in previous studies, public health measures to increase the initiation, duration, and exclusivity of breast feeding seem unlikely to have a major impact on reducing the incidence of atopic diseases. The fact that most atopic outcomes have increased in incidence over the past several decades ${ }^{31}$ simultaneous with the renaissance in breast feeding, strongly suggests that breast feeding does not have a potent protective effect at the population level. Thus, our results underline the importance of seeking other explanations for the recent epidemic of allergy and asthma and of investigating other potential causative factors to develop and test new preventive interventions.

MSK is a senior investigator of the Canadian Institutes of Health Research. RP is a Monat-McPherson career investigator of McGill University, and both he and BM are career investigators (chercheurs-boursiers) of the Fonds de la recherche en santé du Québec

Contributors: MSK, RP, and BM contributed to obtaining funding for this project and to the design, analysis, interpretation, writing, and revision of the manuscript. LM, IV, NB, ZS, ID, and GS contributed both to the design of the study and to the planning, implementation, and monitoring of the field work in Belarus. MSK is the guarantor.

Funding: Grant (MOP No 53155) from the Canadian Institutes of Health Research. The funder had no role in study design, data collection, data analysis, data interpretation, or writing of this manuscript.

Competing interests: None declared.

Ethical approval: The research ethics board of the Montreal Children's Hospital of the McGill University Health Centre approved this project (including the 6.5 year follow-up).

Provenance and peer review: Non-commissioned; externally peer reviewed.

1 Grulee $\mathrm{C}$, Sanford $\mathrm{H}$. The influence of breast and artificial feeding on infantile eczema. J Pediatr 1936;72:411-4.

2 Kramer M. Does breast feeding help protect against atopic disease? Biology, methodology, and a golden jubilee of controversy. J Pediatr 1988:112:181-90.

3 Golding J, Emmett P, Rogers I. Eczema, asthma and allergy. Early Hum Dev 1997;49:S121-30.

4 Gdalevich M, Mimouni D, David M, Mimouni M. Breast-feeding and the onset of atopic dermatitis in childhood: a systematic review and 
meta-analysis of prospective studies. J Am Acad Dermatol 2001;45:520-7.

5 Gdalevich M, Mimouni D, Mimouni M. Breast-feeding and the risk of bronchial asthma in childhood: a systematic review with metaanalysis of prospective studies. J Pediatr 2001;139:261-6.

6 Mimouni Bloch A, Mimouni D, Mimouni M, Gdalevich M. Does breastfeeding protect against allergic rhinitis during childhood? A meta-analysis of prospective studies. Acta Paediatr 2002;91:275-9.

7 Muraro A, Dreborg S, Halken S, Host A, Niggemann B, Aalberse R, et al. Dietary prevention of allergic diseases in infants and small children. Part III: critical review of published peer-reviewed observational and interventional studies and final recommendations. Pediatr Allergy Immunol 2004;15:291-307.

8 Friedman NJ, Zeiger RS. The role of breast-feeding in the development of allergies and asthma. J Allergy Clin Immunol 2005;115:1238-48.

9 Saarinen UM, Kajosaari M. Breastfeeding as prophylaxis against atopic disease: prospective follow-up study until 17 years old. Lancet 1995;346:1065-9.

10 Dell S, To T. Breastfeeding and asthma in young children. Arch Pediatr Adolesc Med 2001;155:1261-5.

11 Kull I, Almqvist C, Lilja G, Pershagen G, Wickman M. Breast-feeding reduces the risk of asthma during the first 4 years of life. J Allergy Clin Immunol 2004;114:755-60.

12 Rothenbacher D, Weyermann M, Beermann C, Brenner H. Breastfeeding, soluble CD14 concentration in breast milk and risk of atopic dermatitis and asthma in early childhood: birth cohort study. Clin Exp Allergy 2005;35:1014-21.

13 Stabell Benn C, Wohlfahrt J, Aaby P, Westergaard T, Benfeldt E, Michaelsen KF, et al. Breastfeeding and risk of atopic dermatitis, by parental history of allergy, during the first 18 months of life. $\mathrm{Am} \mathrm{J}$ Epidemiol 2004;160:217-23.

14 Burgess SW, Dakin CJ, O'Callaghan MJ. Breastfeeding does not increase the risk of asthma at 14 years. Pediatrics 2006;117:787-92.

15 Taylor B, Wadsworth M, Wadsworth J, Peckham C. Changes in the reported prevalence of childhood eczema since the 1939-45 war. Lancet 1984;2:1255-7.

16 Takemura Y, Sakurai Y, Honjo S, Kusakari A, Hara T, Gibo M, et al. Relation between breastfeeding and the prevalence of asthma: the Tokorozawa childhood asthma and pollinosis study. Am J Epidemio 2001;154:115-9.

17 Sears MR, Greene JM, Willan AR, Taylor DR, Flannery EM, Cowan JO, et al. Long-term relation between breastfeeding and development of atopy and asthma in children and young adults: a longitudinal study. Lancet 2002;360:901-7.
18 Purvis DJ, Thompson JMD, Clark PM, Robinson E, Black PN, Wild CJ, et al. Risk factors for atopic dermatitis in New Zealand children at 3 . 5 years of age. BrJ Dermatol 2005;152:742-9.

19 Wegienka G, Ownby DR, Havstad S, Keoki Williams L, Cole Johnson C. Breastfeeding history and childhood allergic status in a prospective birth cohort. Ann Allergy Asthma Immunol 2006;97:78-83.

20 Wright AL, Holberg CJ, Taussig LM, Martinez FD. Factors influencing the relation of infant feeding to asthma and recurrent wheeze in childhood. Thorax 2001;56:192-7.

21 Lowe AJ, Carlin JB, Bennett CM, Abramson MJ, Hosking CS, Hill DJ, et al. Atopic disease and breast-feeding-cause or consequence? J Allergy Clin Immunol 2006;117:682-7.

22 Kramer MS, Chalmers B, Hodnett ED, Sevkovskaya Z, Dzikovich I, Shapiro S, et al. Promotion of breastfeeding intervention trial (PROBIT): a randomized trial in the Republic of Belarus. JAMA 2001;285:413-20.

23 WHO/UNICEF. Protecting, promoting and supporting breastfeeding: the special role of maternity services. Geneva: World Health Organization, 1989.

24 Campbell MK, Elbourne DR, Altman DG, for the CONSORT Group. CONSORT statement: extension to cluster randomised trials. $B M$ 2004;328:702-8.

25 Asher MI, Keil U, Anderson HR, Beasley R, Crane J, Martinez F, et al. International study of asthma and allergies in childhood (ISAAC): rationale and methods. Eur Respir J 1995;8:483-91.

26 Breslow NE, Clayton DG. Approximate inference in generalized linear mixed models. J Am Stat Assoc 1993;88:9-25.

27 Oddy WH, Holt PG, Sly PD, Read AW, Landau LI, Stanley F), et al. Association between breast feeding and asthma in 6 year old children: findings of a prospective birth cohort study. BMJ 1999;319:815-9.

28 International Study of Asthma and Allergies in Childhood (ISAAC) Steering Committee. Worldwide variation in prevalence of symptom of asthma, allergic rhinoconjunctivitis, and atopic eczema: ISAAC. Lancet 1998;351:1225-32.

29 Oppenheimer J, Nelson HS. Skin testing. Ann Allergy Asthma Immunol 2006;96(suppl 1):S6-12.

30 Ronchetti R, Villa MP, Bohmerova Z, Martella S, Falasca C, Barreto M, et al. Skin reactivity to histamine and codeine in unselected 9-yearold children from Italy, Poland and Libya. Int Arch Allergy Immunol 2004;135:136-42.

31 Eder W, Ege MJ, von Mutius E. The asthma epidemic. N Engl J Med 2006;355:2226-35.

Accepted: 27 July 2007 